\title{
Aspergillus sellar abscess: Case report and review of the literature
}

\author{
Li Hao, Chen Jing, Cai Bowen, He Min, You Chao \\ Department of Neurosurgery, West China Hospital of Sichuan University, 37 Guo Xue Xiang, Wu Hou District, Chengdu - 610041 , China.
}

\begin{abstract}
Aspergillus sellar abscess is a very rare form of fungal infections of the central nervous system (CNS). In this report, we describe the successful treatment of a patient with aspergillus sellar abscess. A 65-year-old woman presented with headache, nasal discharge and decreased visual acuity. The diagnosis of sellar mass was made on the basis of magnetic resonance imaging (MRI) examination. The computed tomography (CT) scan revealed sellar enlargement and sellar floor bony destruction. After hospitalization the patient underwent transsphenoidal surgery. Histopathological examination of the sellar mass revealed aspergillosis. Postoperatively, amphotericine-B and itraconazole therapy was started. During a six-month follow-up, the patient's headache and inertia disappeared, visual acuity improved. Aspergillus sellar abscess must be considered in the differential diagnosis of a sellar mass. The correct diagnosis of pituitary aspergillosis can only be achieved by histopathological examination. Surgical intervention and antifungal therapy should be considered the optimal treatment.
\end{abstract}

Key words: Amphotericine-B, aspergillus, sellar abscess, transsphenoidal surgery

Aspergillus sellar abscess, which is a rare form of central nervous system (CNS) fungal infections, is usually misdiagnosed because its presentation is similar to that of a pituitary adenoma. Although it is benign and curable, a delay in the diagnosis and treatment may lead to significant morbidity. Surgical resection allows for a histopathological diagnosis, which is necessary for any sellar mass identified by magnetic resonance imaging (MRI).

\section{Case Report}

A 65-year-old woman was admitted to our hospital with five months of headache, nasal discharge, two months of decreased visual acuity, with a deteriorating condition for a week. She complained of bilateral frontal-temporal drill pain, and inertia. The diagnosis of sellar mass was made on the basis of MRI examination. Her medical history was insignificant for diabetes, malignancies, drug abuse, blood transfusions, meningitis and head trauma. Physical examination was normal and the patient was afebrile. The neurological examination revealed bilateral papilledema and bitemporal hemianopsia and impairment of visual acuity. The MRI revealed a sellar suprasellar mass with a dimension of $2.5 \mathrm{~cm} \times 2.5$ $\mathrm{cm} \times 2.5 \mathrm{~cm}$, iso-intense on T1-weighted images with peripheral hyperdensity. On T2-weighted images the central part of the mass was hyperintense although peripheral iso-intensity was present. Contrast enhanced MRI examination also showed that the sellar mass had a peripheral contrast enhanced rim [Figures 1, 2]. The computed tomography (CT) scan revealed sellar enlargement and sellar floor bony destruction

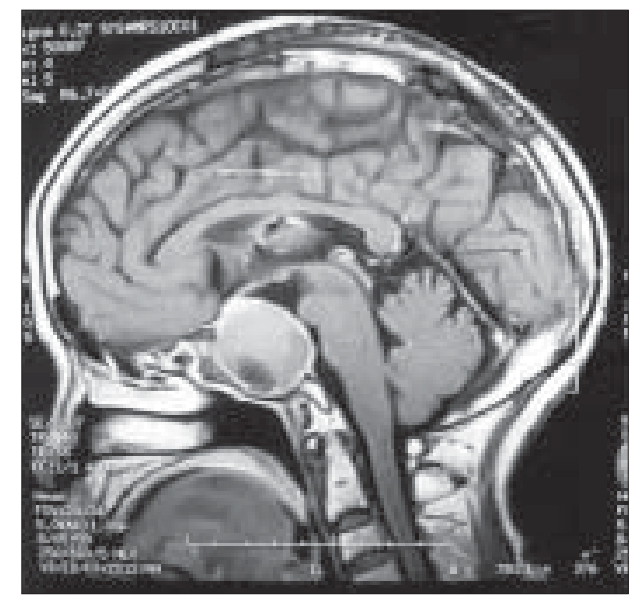

Figure 1: Preoperative gadolinium enhanced T1-weighted MRI coronal scan showing sellar suprasellar mass with peripheral contrast enhancement 


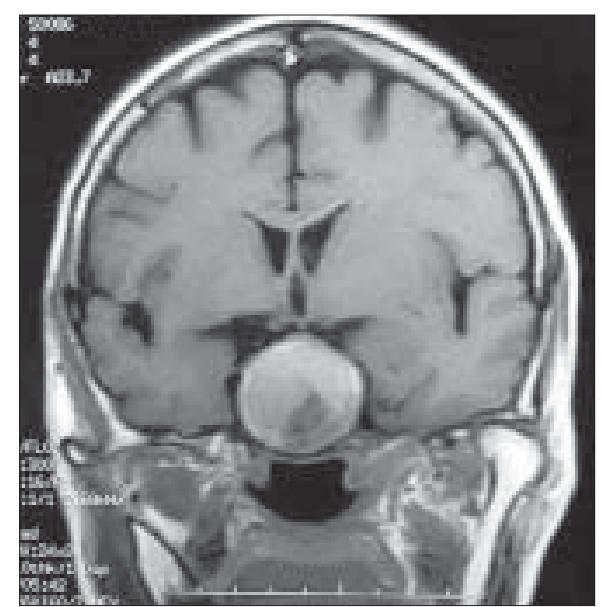

Figure 2: Preoperative gadolinium enhanced T1-weighted MRI coronal scan showing sellar suprasellar mass with peripheral contrast enhancement. Marked elevation of the optic chiasma and thickened sphenoid sinus mucosa suggesting sinusitis can also be seen

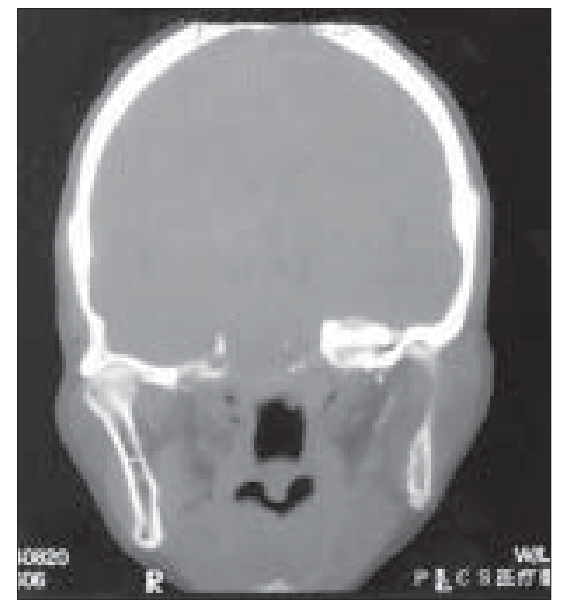

Figure 3: The CT scan revealed sellar enlargement and sellar floor bony destruction

[Figure 3]. Endocrinological tests showed depressed levels of adrenocorticotrophic hormone (ACTH), growth hormone $(\mathrm{GH})$, prolactine (PRL), thyrotropicstimulating hormone (TSH), gonadotrophines. After hospitalization the patient underwent transsphenoidal surgery. When the sphenoid sinus was opened, the mucosa, which cultured optimally on Sabourad's agar demonstrates characteristic conidiophores, was found to be thickened and purulent material was seen, and blood and cerebrospinal fluid cultures negative. The sellar floor bony was eroded. A necrotic sellar mass was removed through the microscope. The diaphragma sellae was intact and not opened. The sellar cavity and sphenoid sinus were irrigated with gentamycin solution. At the end of the operation we aerated the infected sphenoid sinus. Histopathological examination of the sellar mass resulted in the diagnosis of aspergillosis. After the operation, amphotericine-B $(25 \mathrm{mg} / \mathrm{d})$ and itraconazole $(800 \mathrm{mg} / \mathrm{d})$ therapy was started. During a 6-month follow-up, the patient's headache and inertia disappeared, visual acuity improved.

\section{Discussion}

Aspergillus is a fungal organism which has lots of species and a few of them are pathogenic. ${ }^{[1]}$ Aspergilli are opportunistic pathogens which are ubiquitous fungi found in soil and organic materials and can establish saprophytic growth within the respiratory tract after inhalation. The organism can become pathogenic under immune suppression, granulocytopenia, diabetes mellitus or prolonged hospitalization. Central nervous system (CNS) aspergillosis presents as meningitis, encephalitis, brain abscesses, subdural abscesses, mycotic arteritis or sellar abscess. ${ }^{[2]}$ Aspergillus can reach the CNS by three different routes. The first is by hematogenous spread from a remote extracranial focus, usually the lung. The second is by extension from a contiguous extracranial location which is often the paranasal sinus or the nasal cavity. The third is direct introduction of aspergillus by a neurosurgical procedure iatrogenically. ${ }^{[3]}$ In our case, the patient presented with nasal cavity and sphenoid sinus infection inflammation, the CT scan showed sellar floor bony destruction, therefore, aspergillus infection probably spread from the nasal sinus.

Diagnosis of CNS aspergillosis is difficult because the presenting symptoms in these patients are extremely identical to the symptoms in patients with pituitary adenomas. Headaches, visual disturbances and endocrine failure are the common symptoms and signs. ${ }^{[4]}$ On CT usually sellar enlargement and a lowdensity sellar mass with contrast enhanced rim is seen. Magnetic resonance imaging is the best examination in the radiological evaluation of a sellar abscess..$^{[5,6]} \mathrm{A}$ sellar abscess usually appears as a hypo-iso-intense sellar mass on T1-weighted sequences and as a hyperintense mass on T2-weighted sequences. A peripheral contrast enhancing rim is usually present. In our case, plain MRI findings suggested a pituitary adenoma, although contrast enhanced rim of the mass and nasal cavity infection findings led us to the preoperative diagnosis of sellar abscess.

However, in our case the diagnosis of sellar aspergillosis was made as a result of histopathological examination because clinical and radiological findings were not specific.

The treatment of the sellar abscess is surgical resection via a transsphenoidal approach, and a craniotomy increases the risk of contaminating subarachnoid spaces and causing meningo-encephalitis. Transsphenoidal approach is also dangerous because it is absolutely close to the border of the subarachnoid space. We must rigorously avoid even microtraumas of the diaphragma sellae and keep it intact, because of 
the risk of a subarachnoid spread with aspergillus, such infections are lethal in most cases. In the nasal respectively sphenoid phase, at the end of the operation the neurosurgeon has to aerate the infected sphenoid sinus because a closure of the situs leads to a recurrent infection. Antibiotic treatment alone is not enough to save the infected region and to prevent a re-infection.

Immediately after the histopathological diagnosis, the patients with aspergillus sellar abscess must be treated intravenously with amphotericine-B or combined therapy with amphotericine-B and flucytosine or itraconazole. In India, Kshirsagar et al had developed liposomal amphotericin, to patient-worthy, sterile pyrogen free preparations. The same group studied different dosage regimens of liposomal amphotericin in Aspergillus model and found that liposomal amphotericin was more effective than equal dose of free amphotericin-B. Subsequent clinical trials with Indian liposomal amphotericin-B (Fungisome ${ }^{\mathrm{TM}}$ ) have demonstrated its safety and efficacy in the treatment of CNS aspergilloma. ${ }^{[7]}$ In focal CNS aspergillosis total cure can be achieved in approximately $30 \%$ of the cases by surgical resection and intensive antifungal therapy.

In conclusion, aspergillus sellar abscess should be considered in the differential diagnosis of a pituitary mass. The correct diagnosis of sellar aspergillosis can only be achieved by histopathological examination of tissue obtained at surgery. The optimal treatment is a combination of surgical resection and intensive antifungal therapy.

\section{References}

1 Parker KM, Nicholson JK, Cezayirli RC, Biggs PJ. Aspergillosis of the sphenoid sinus: Presentation as a pituitary mass and postoperative Gallium-67 imaging. Surg Neurol 1996;45:354-8.

2 Endo T, Tominaga T, Konno H, Yoshimoto T. Fatal subarachnoid hemorrhage, with brainstem and cerebellar infarction, caused by Aspergillus infection after cerebral aneurysm surgery: Case report. Neurosurg 2002;50:1147-51.

3 Endo T, Numagami Y, Jokura H, Ikeda H, Shirane R, Yoshimoto T. Aspergillus parasellar abscess mimicking radiation-induced neuropathy: Case report. Surg Neurol 2001;56:195-200.

4 Vates GE, Berger MS, Wilson CB. Diagnosis and management of pituitary abscess: A review of twenty-four cases. J Neurosurg 2001;95:233-41.

5 Iplikcioglu AC, Bek S, Bikmaz K, Ceylan D, Gökduman CA. Aspergillus pituitary abscess. Acta Neurochir (Wien) 2004;146:521-4.

6 Alapatt JP, Kutty RK, Gopi PP, Challissery J. Middle and posterior fossa aspergilloma. Surg Neurol 2006;66:75-9.

7 Bodhe PV, Kotwani RN, Kirodian BG, Kshirsagar NA, Pandya SK. Open label, randomized, comparative Phase III safety and efficacy study with conventional amphotericin $\mathrm{B}$ and liposomal amphotericin $\mathrm{B}$ in patients with systemic fungal infection. J Assoc Physicians India 2002;50: 662-70.

Accepted on 28-03-2008

Source of Support: Nil, Conflict of Interest: None declared. 